\title{
THE FEATURES OF THE NON-EQUILIBRIUM EQUATION OF STATE IN HEAVY-ION COLLISIONS AT INTERMEDIATE ENERGIES
}

\author{
D’yachenko A.T. ${ }^{1}$, Gridnev K.A. ${ }^{2}$, Mitropolsky I.A. ${ }^{3}$ \\ ${ }^{1}$ Petersburg State Transport University, Russia; ${ }^{2}$ Saint Petersburg State University, Russia; \\ ${ }^{3}$ Russian Research Center "Kurchatov Institute", \\ B.P.Konstantinov Petersburg Nuclear Physics Institute, Gatchina, Russia \\ E-mail: dyachenko_a@mail.ru
}

In the development of the approach in [1] for the description of heavy-ion collisions, we proposed to use the method of moments for solving the kinetic equation, which gives, for the first moments with weights $1, \vec{p}, p^{2}$, equations of the hydrodynamic type, allowing us to find the distribution function of the nucleons, $f(\vec{r}, \vec{p}, t)$ (where $\vec{r}$ is the spatial coordinate, $\vec{p}$ is the momentum, and $t$ is the time). At the same time, we used a non-equilibrium equation of state to describe the hydrodynamic evolution of a system of the «hot spot» type, according to which $f(\vec{r}, \vec{p}, t)$ is associated with an equilibrium component, $f_{0}(\vec{r}, \vec{p}, t)$, and a non-equilibrium component $f_{1}(\vec{r}, \vec{p}, t)$ :

$$
f(\vec{r}, \vec{p}, t)=f_{1} \cdot q+f_{0} \cdot(1-q),
$$

where $q$ is the relaxation factor. Here, $f_{1}(\vec{r}, \vec{p}, t)$ is given in the form of a Fermi-ellipsoid in the momentum space, which is a convenient parameterization of the excitations in the Fermi-liquid theory, and $f_{0}(\vec{r}, \vec{p}, t)$ is given as the equilibrium Fermi-sphere. In this approach, the account of the non-equilibrium component has led to a successful description of the heavy-ion collision dynamics, in particular to the description of the energy spectra of protons emitted at various angles [1-4].

In the present paper we develop a method for the calculation of the factor $q$ by taking the moment from a kinetic equation with weight $p_{x}^{2}-\left(p_{y}^{2}+p_{z}^{2}\right) / 2$ that determines the degree of anisotropy of the distribution function, $f(\vec{r}, \vec{p}, t)$. We found that $q=1$ on the shock wave during the compression stage in the energy range $\sim 10-100 \mathrm{MeV} /$ nucleon and that $q$ decreases with increasing energy, which results in an increase of the isotropy of the function $f(\vec{r}, \vec{p}, t)$ at higher intermediate energies, in accordance with experimental data on the proton spectra.

1. A.T.D'yachenko, K.A.Gridnev // Proc. of the 63rd Int. Conf. "Nucleus 2013". Book of Abstracts. October 8-12, 2013. Moscow. St. Petersburg. 2013. P.207.

2. A.T.D’yachenko // Phys. Atom. Nucl. 1994. V.57. P.1930.

3. A.T.D’yachenko, K.A.Gridnev // Bull. Russ. Acad. Sci. Phys. 2013. V.77. P.857.

4. A.T.D’yachenko, K.A.Gridnev, W.Greiner // J. Phys. G. Nucl. Part. Phys. 2013. V.40. 085101. 